\title{
miR-206 inhibits FN1 expression and proliferation and promotes apoptosis of rat type II alveolar epithelial cells
}

\author{
JUN DUAN, XIAOYING ZHANG, SHENG ZHANG, SHAODONG HUA and ZHICHUN FENG \\ Department of Pediatrics, BaYi Children's Hospital Affiliated to Clinical Medical College \\ in Beijing Military General Hospital of Southern Medical University, Beijing 100700, P.R. China
}

Received November 24, 2015; Accepted December 19, 2016

DOI: $10.3892 / e t m .2017 .4430$

\begin{abstract}
Bronchopulmonary dysplasia (BPD) is a syndrome of respiratory distress caused by chronic lung injury, primarily in preterm infants. miR-206 and fibronectin 1 (FN1) are associated with the development of BPD. The present study used rat type II alveolar epithelial cells (AECII) to investigate the underlying mechanisms of BPD. AECII were isolated using a primary cell culture prior to alkaline phosphatase staining and immunofluorescence of surfactant protein C (SP-C). These were used to verify the presence of AECII. AECII were then divided into four groups, which were transfected with four different plasmids. Reverse transcription-quantitative polymerase chain reaction (RT-qPCR) was performed to determine the relative expression of miR-206 in the each group. The gene and protein expression level of FN1 was detected by RT-qPCR and immunofluorescence. The proliferation of AECII in each of the four groups was evaluated using an MTT assay $48 \mathrm{~h}$ following transfection. The percentage of apoptotic cells was determined by flow cytometric analysis. The present study demonstrated that upregulation of miR-206 decreased the expression of FN1 $(\mathrm{P}<0.05)$ and low levels of miR-206 led to increased expression of FN1 $(\mathrm{P}<0.05)$ in AECII. Furthermore, the forced expression of miR-206 suppressed proliferation and promoted apoptosis of AECII while downregulation of miR-206 had the opposite effect $(\mathrm{P}<0.05)$. The results of the
\end{abstract}

Correspondence to: Dr Zhichun Feng or Dr Xiaoying Zhang, Department of Pediatrics, BaYi Children's Hospital Affiliated to Clinical Medical College in Beijing Military General Hospital of Southern Medical University, 5 Nanmencang Road, Dongcheng, Beijing 100700, P.R. China

E-mail: fengzc81@163.com

E-mail: xiaoyingzhang79@126.com

Abbreviations: AECII, type II alveolar epithelial cells; BPD, bronchopulmonary dysplasia; ECM, extracellular matrix; miRNAs, microRNAs; MTT,3-(4,5-dimethylthiazol-2-yl)-2,5-diphenyltetrazolium; RT-qPCR, reverse transcription-quantitative polymerase chain reaction; TGF- $\beta 1$, transforming growth factor- $\beta 1$; FN1, fibronectin 1

Key words: bronchopulmonary dysplasia, fibronectin 1, lung injury, rat type II alveolar epithelial cells, microRNA-206 current study provide valuable insights into the prevention of BPD and suggest that miR-206 may be used as a potential molecular target for BPD therapy in the future.

\section{Introduction}

Bronchopulmonary dysplasia (BPD) is a common chronic lung disease in preterm infants who require assisted ventilation $(1,2)$. BPD is characterized by impaired alveolarization, a primary cause of mortality in premature infants (3). A number of of genetic and environmental factors have been identified to be responsible for development of BPD (4-6). However, the complete mechanism of BPD remains unknown and further studies are required to identify novel mechanisms involved in the process.

Fibronectin 1 (FN1), a component of the extracellular matrix (ECM), is widely distributed in blood vessel and smooth-muscle cell layers (7). Abnormal expression of FN1 has been identified in a number of human diseases, including cancers (8). FN1 may also interact with other ECM proteins, which may in turn serve important roles in the development of $\operatorname{BPD}(9,10)$.

microRNAs (miRNAs or miRs) were first identified in the early 1990s $(11,12)$. miRNAs regulate gene expression post-transcriptionally and serve essential roles in a wide range of biological functions, including cell proliferation, apoptosis and differentiation (13-16). A number of previous investigations indicated that miRNAs are associated with the process of fetal lung maturation and metabolism of pulmonary surfactant $(17,18)$. Previous studies have demonstrated the important role of miRNAs in the development of BPD $(19,20)$. It has also been indicated that miR-206 is downregulated in mice modeling BPD and BPD in human patients (21).

BPD is a chronic disease characterized by disruption of the alveolar and microvascular development of the peripheral lung. The regeneration or stimulation of type II alveolar epithelial (AECII) cell growth has been the focus of studies aiming to regenerate the lung $(1,3,7)$. Therefore, AECII is critical for development and thus the treatment of BPD $(21,22)$. Due to the importance of AECII, it was selected as the experimental model to elucidate the mechanism of miR-206 affecting BPD in the present study. Using a primary cell culture of AECII allows the possible effects of miR-206 on AECII to be studied. Reverse transcription-quantitative polymerase chain reaction (RT-qPCR) 
and immunofluorescence were performed to study the effect of miR-206 on FN1. Different levels of miR-206 expression indicated proliferation and apoptosis of AECII. The present study provides insight into the function of AECII and improves the understanding of its role in the development of BPD.

\section{Materials and methods}

Cell isolation and culture. Four two-month old SpragueDawley female rats (200-250 g) were obtained from Beijing Military General Hospital of Southern Medical University (Beijing, China). Rats were housed in cages at the temperature of $22 \pm 2^{\circ} \mathrm{C}$ and $40 \pm 5 \%$ humidity under a 12-h light/dark cycle and received a standard diet and water ad libitum. Following anesthetization with an injection of pentobarbital (0.2 ml/100 g; Beijing Solarbio Science \& Technology Co. Ltd., Beijing, China), rat lungs were obtained by surgical resection and digested by trypsin (Gibco; Thermo Fisher Scientific, Inc., Grand Island, NY, USA) through the trachea (18). Lung cells were transferred to dishes coated with rat immunoglobulin (Ig) G $(500 \mu \mathrm{g} / \mathrm{ml}$; SP032; Beijing Solarbio Science $\&$ Technology Co., Ltd.) on the basis of the different adherence abilities of cells. AECII in the supernatant was collected by pipette and cultured in Dulbecco's modified Eagle's medium (DMEM) supplemented with 10\% fetal bovine serum (Gibco; Thermo Fisher Scientific, Inc.) in an incubator at $37^{\circ} \mathrm{C}$ with $5 \%$ $\mathrm{CO}_{2}$ for $24 \mathrm{~h}$.

Identification of AECII. For the alkaline phosphatase (BCIP/NBT) staining, cells were fixed in $4 \%$ formaldehyde (Beijing Solarbio Science \& Technology, Co., Ltd.) at room temperature for $10 \mathrm{~min}$. Following washing with phosphate-buffered saline (PBS) three times, cells were incubated with $3 \mathrm{ml}$ BCIP/NBT solution (Beyotime Institute of Biotechnology, Shanghai, China) for $10 \mathrm{~min}$ at room temperature. Cells were then rinsed with water, blotted dry and imaged using a C1si inverted microscope (Nikon Corporation, Tokyo, Japan).

Immunofluorescence of surfactant protein C (SP-C) was used to verify AECII. SP-C rabbit anti-mouse polyclonal antibody (1:100; sc-13979; Santa Cruz Biotechnology, Inc., Dallas, TX, USA) was incubated at $4^{\circ} \mathrm{C}$ for $15 \mathrm{~h}$, then TRITC-conjugated goat anti-rabbit polyclonal antibody (1:200; 85851; Jackson ImmunoResearch Laboratories, Inc., West Grove, BA, USA) was added and incubated at $37^{\circ} \mathrm{C}$ for $1 \mathrm{~h}$. Hoechst 33258 (Beyotime Institute of Biotechnology) was added to label the nuclei. A confocal microscope (Nikon Corporation) was used to observe the cells. The present study was approved by the Ethics Committee of Beijing Military General Hospital (Beijing, China) and conformed to the guidelines for the Care and Use of Laboratory Animals of the National Institutes of Health (23).

Plasmid synthesis. All plasmids were synthesized by Shanghai GenePharma, Co., Ltd. (Shanghai, China), including the overexpression plasmid for miR-206 (pcmv-206), the negative control (pcmv-nc), miRNA inhibitors for miR-206 (pgpu6-206) and inhibitor negative control (pgpu6-nc).

Transient transfection. AECII were randomly divided into four groups. Group 206 were transfected with pcmv-206, group nc were transfected with pcmv-nc, group 206-i were transfected with pgpu6-206 and group nc-i were transfected with pgpu6-nc. Cells were transfected by Lipofectamine ${ }^{\circledR} 2000$ (Invitrogen; Thermo Fisher Scientific, Inc.) according to manufacturer protocol. When grown to $80-90 \%$ confluence $24 \mathrm{~h}$ later, AECII were transfected with the four plasmids. AECII were harvested at $48 \mathrm{~h}$ following transfection for subsequent studies. Transfection experiments were completed three times.

RNA extraction and RT-qPCR. RT-qPCR was performed to detect the gene expression of miR-206 and FN1. Total RNA of AECII was extracted using TRIzol ${ }^{\circledR}$ (Invitrogen; Thermo Fisher Scientific, Inc.) according to a standard protocol (7). Then cDNA was synthesized by reverse-transcription reaction. RT-qPCR was conducted using THUNDERBIRD ${ }^{\circledR} \mathrm{SYBR}^{\circledR}$ qPCR mix (Toyobo, Co., Ltd., Osaka, Japan). The ABI Prism ${ }^{\circledR} 7000$ Sequence Detection system (Applied Biosystems; Thermo Fisher Scientific, Inc.) was used to analyze the product. The PCR primers were designed using Primer Premier 5.0 (PREMIER Biosoft, Inc., Paolo Alto, CA, USA) complementary to rat miR-206 and FN1 cDNA sequences (Sangon Biotech Co., Ltd., Shanghai, China). The primer sequences for RT-qPCR were as follows: miR-206 forward, 5'-GGGTGGA ATGTAAGGAAGT-3' and reverse, 5'-TGCGTGTCGTGGA GTC-3'; U6 forward, 5'-GCTTCGGCAGCACATATACTAA AAT-3' and reverse, 5'-CGCTTCACGAATTTGCGTGT CAT-3; FN1 forward, 5'-CTCGCTTTGACTTCACCACCA-3' and reverse, 5'-TCTCCTTCCTCGCTCAGTTCGTACT-3'; $\beta$-actin forward, 5'-TGGAATCCTGTGGCATCCATGAAAC-3' and reverse, 5'-TAAAACGCAGCTCAGTAACAGTCCG-3'. The ABI Prism ${ }^{\circledR} 7000$ Sequence Detection system program was as follows: $95^{\circ} \mathrm{C}$ initial denaturation for $5 \mathrm{~min}$, followed by 40 cycles of denaturation at $95^{\circ} \mathrm{C}$ for $10 \mathrm{sec}$, annealing at $59^{\circ} \mathrm{C}$ for $15 \mathrm{sec}$ and elongation at $72^{\circ} \mathrm{C}$ for $33 \mathrm{sec}$. Relative expressions of miR-206 and FN1 were analyzed with normalization against $\mathrm{U} 6$ and $\beta$-actin values respectively using the $2^{-\Delta \Delta \mathrm{Cq}}$ method described by Livak and Schmittgen (24). All reactions were performed three times.

Immunofluorescence of FN1. Four slides of AECII were incubated with a polyclonal rabbit anti-mouse FN1 antibody (1:100; 15613-1-AP; Proteintech, Rosemont, IL, USA) at $4^{\circ} \mathrm{C}$ overnight. A goat anti-rabbit polyclonal $\mathrm{IgG}$ conjugated with TRITC (85851; Jackson ImmunoResearch Laboratories, Inc.) was added as the secondary antibody $(1: 200)$ at $37^{\circ} \mathrm{C}$ for $1 \mathrm{~h}$. The cellular nuclei were stained with Hoechst 33258 (Beyotime Institute of Biotechnology). A confocal microscope (Nikon Corporation) was used to image the immunofluorescence.

Proliferation assays. To study the effect of miR-206 on the proliferation of AECII, 4x104 cells were seeded into 96-well culture plate and transfected with one of the four plasmids. The cell viability was analyzed using an MTT assay at $48 \mathrm{~h}$ after transfection. First, $20 \mu \mathrm{l}$ MTT (Sigma-Aldrich; Merck KGaA, Darmstadt, Germany) was added to the 96-well plate and incubated at $37^{\circ} \mathrm{C}$ for $4 \mathrm{~h}$ in the dark. Then, the supernatant was carefully discarded and $150 \mu$ l dimethyl sulfoxide (Sigma-Aldrich; Merck KGaA) was added to the plate at room temperature for $20 \mathrm{~min}$. The MTT optical density value was measured at $492 \mathrm{~nm}$ using a microplate reader (Bio-Rad 

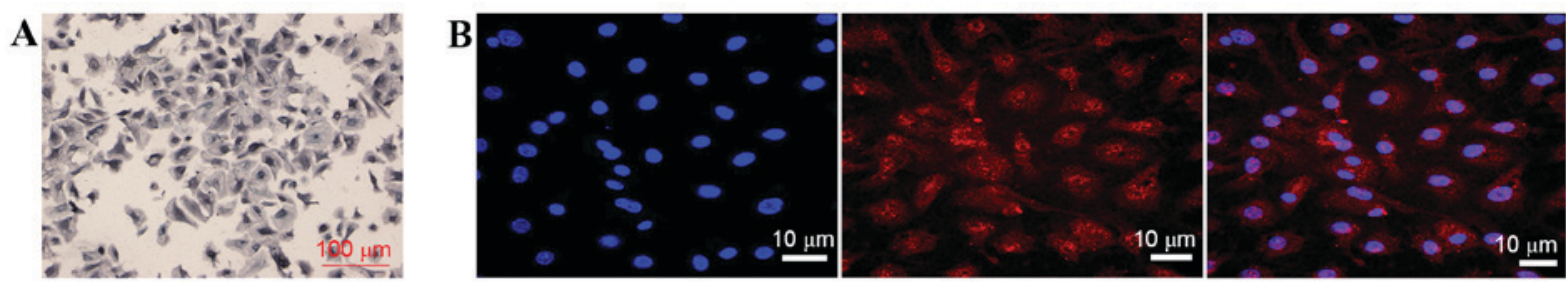

Figure 1. Identification of AECII following alkaline phosphatase staining and immunofluorescence of SP-C. (A) Alkaline phosphatase staining of AECII. Positive staining indicated by grayish staining in cells. (B) Immunofluorescence of surfactant protein SP-C in AECII. A strong red fluorescence signal indicated by SP-C. AECII, type II alveolar epithelial cells; SP-C, surfactant protein C.

Laboratories, Inc., Hercules, CA, USA). The experiment was repeated three times.

Apoptosis assay. A total of $1 \times 10^{6}$ AECII were plated into six-well plates and transfected with four plasmids using Lipofectamine $^{\circledR}$ 2000. Apoptosis inducer, cisplatin (Beyotime Institute of Biotechnology) was also added to the culture. Cells were collected $48 \mathrm{~h}$ following transfection and stained with Annexin V and propidium iodide (APT750; Merck Millipore, Billerica, MA, USA) according to manufacturer protocol. The apoptotic percentage was determined by flow cytometric analysis (Beckman Coulter, Inc., Brea, CA, USA). All these procedures were repeated in triplicate.

Statistical analysis. Experiments were repeated three times and data were presented as the mean \pm standard deviation. SPSS 13.0 software (SPSS, Inc., Chicago, IL, USA) was used for statistical analysis. Differences between two groups were calculated with the two-tailed $t$-test. $\mathrm{P}<0.05$ was considered to represent statistically significant differences.

\section{Results}

Identification of AECII. AECII have alkaline phosphatase activity however, other lung cells including alveolar macrophages lack phosphatase activity. Therefore, alkaline phosphatase activity was assessed to distinguish AECII from other cells. Positive staining was indicated by grey staining in the cells (Fig. 1A).

AECII secretes pulmonary surfactants. SP-C, the only AECII-specific surfactant, was also used to identify AECII (Fig. 1B). The red fluorescence in Fig. 1B indicates SP-C, which was located in the cytoplasm. The blue fluorescence indicates the nuclei. The results indicated that the isolation of AECII was successful.

Expression of miR-206 in AECII. To investigate the function of miR-206, exogenous miR-206 plasmids, inhibitors and control plasmids were assessed. To increase the expression of miR-206, pcmv-206 was transfected into AECII. To maintain the stable expression of miR-206 in AECII, pcmv-nc was transfected into AECII. AECII were harvested $48 \mathrm{~h}$ following transfection and the expression of miR-206 was determined using RT-qPCR. The level of miR-206 in group 206 significantly increased by $\sim 30$-fold compared with group nc ( $\mathrm{P}<0.05$; Fig. 2A).

To downregulate the expression of miR-206, pgpu6-206 was transfected into AECII and pgpu6-nc was transfected into AECII as controls. In group 206-i, the expression of miR-206
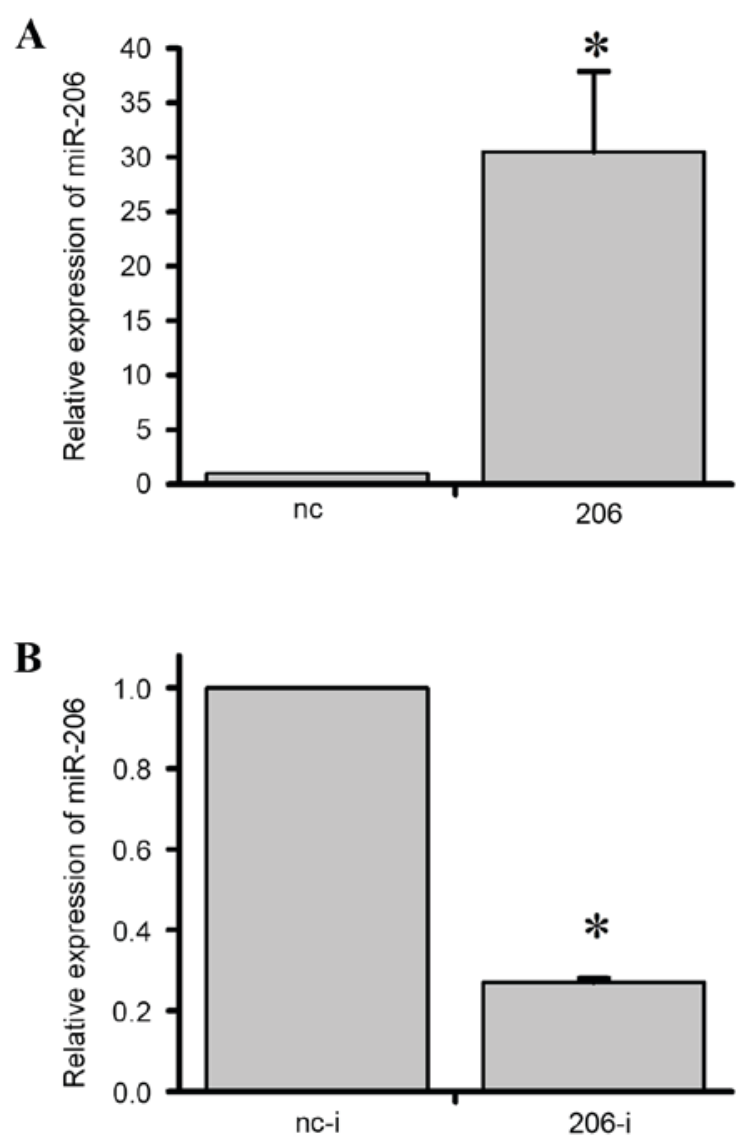

Figure 2. Relative expression of miR-206 in four groups. (A) Relative expression of miR-206 in group 206 increased compared with nc group. (B) Relative expression of miR-206 in group 206-i decreased compared with group nc-i. " $\mathrm{P}<0.05$. miR-206, micro RNA 206; nc, group transfected with pcmv-nc; 206-i, group transfected with pgpu6-206; nc-i, group transfected with pgpu6-nc.

significantly reduced compared with group nc-i $(\mathrm{P}<0.05$; Fig. 2B). These data indicated that the four different plasmids transfected AECII successfully, which provides important basis for further study.

MiR-206 inhibited the expression of FN1 in AECII. To determine whether the synthesis of FN1 is affected by miR-206 in AECII, RT-qPCR was completed to detect the relative expression of FN1 at a gene level. As presented in Fig. 3A, FN1 mRNA expression in group 206 decreased by $80 \%$ compared with the control group $(\mathrm{P}<0.05)$. By contrast, low expression of miR-206 caused an increase in FN1 expression by $\sim$ seven-fold compared with control group $(\mathrm{P}<0.05$; Fig. $3 \mathrm{~A})$. 
A

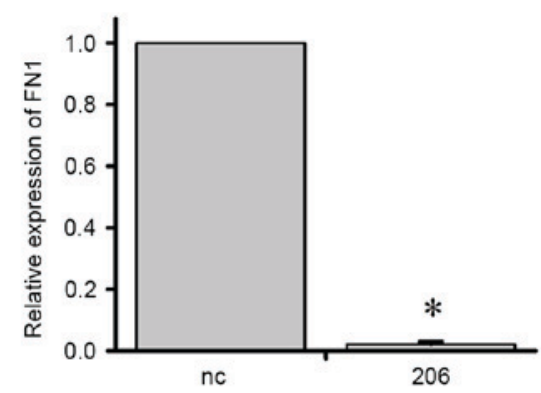

B

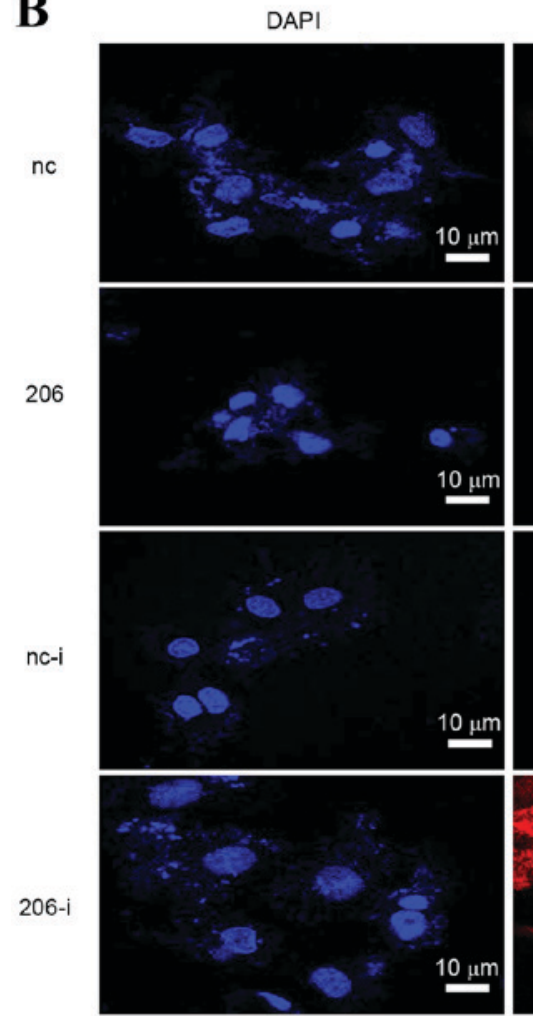

FN1

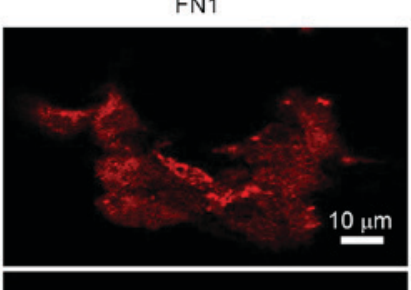

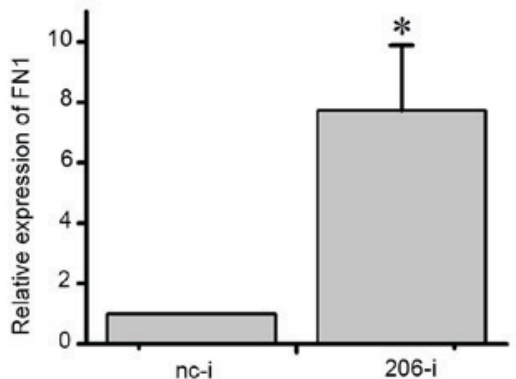

Merge

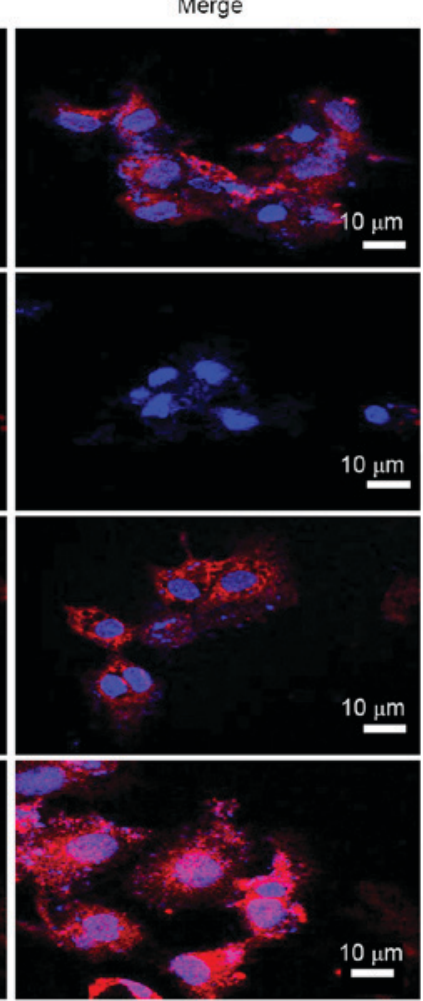

Figure 3. Relative expression of FN1 in four groups. (A) Detection of FN1 mRNA expression in AECII was performed by reverse transcription-quantitative polymerase chain reaction ("P<0.05). (B) Detection of FN1 protein expression in AECII performed by immunofluorescence. N1, fibronectin 1; AECII, type II alveolar epithelial cells; nc, group transfected with pcmv-nc; nc-i, group transfected with pgpu6-206; 206, group transfected with pcmv-206; 206-i, group transfected with pgpu6-206.

To confirm the effect of miR-206 on protein level, immunofluorescence was used to detect the level of FN1 protein in AECII. Group 206 demonstrated a weak signal of red fluorescence of FN1 and 206-i group demonstrated a strong signal of red fluorescence of FN1 (Fig. 3B). This was consistent with the effect of miR-206 on the expression of genes.

In the present study, four different plasmids were transfected into AECII to investigate the effect of miR-206 on FN1 in AECII. The results indicated that FN1 may be negatively regulated by miR-206 in AECII thus, suggesting that FN1 may be involved in the process of BPD.

MiR-206 inhibits proliferation and promotes apoptosis of AECII. MTT assay was performed to assess whether miR-206 had an effect on the proliferation of AECII. Following transfection with pcmv-206 (group 206), the proliferation capability of AECII decreased by $19 \%$ compared with control group $(\mathrm{P}<0.05)$. When transfected with pgpu6-206 (group 206-i), the proliferation capability of cells increased compared with cells transfected with pgpu6-nc by $23 \%(\mathrm{P}<0.05$; Fig. 4A). The flow cytometry assay results indicated that the apoptotic rate increased in the 206 group and reduced in group 206-i compared with the respective control groups ( $\mathrm{P}<0.05$; Fig. 4B). The findings suggest that miR-206 inhibited the proliferation and promoted the apoptosis of AECII.

\section{Discussion}

Although much remains to be fully studied, previous investigations suggest that miRNAs serve an important role by controlling the function of various genes involved in cell functions (13-15). miRNAs regulate gene expression post-transcriptionally and inhibit the expression of the target genes $(13,25)$. As a member of the miRNA family, miR-206 regulates the translation of target mRNAs based on sequence complementarity $(12,26)$. Previous studies have suggested that miR-206 is associated with a variety of diseases (26-29). Lewis et al (30) reported that miR-206 was associated with 
A

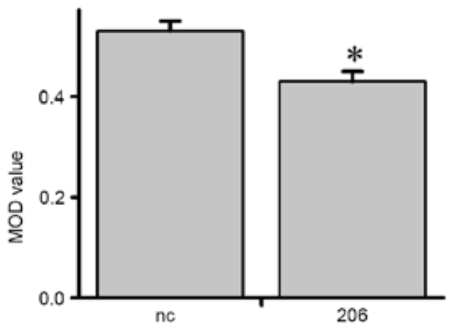

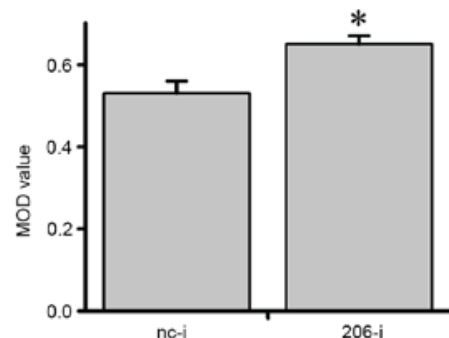

B
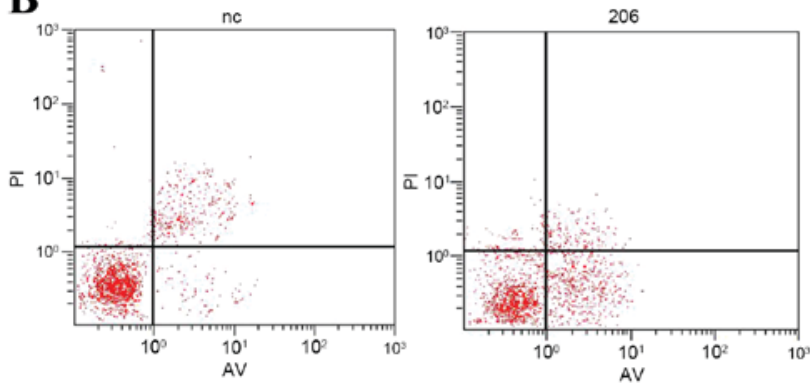

$206-i$
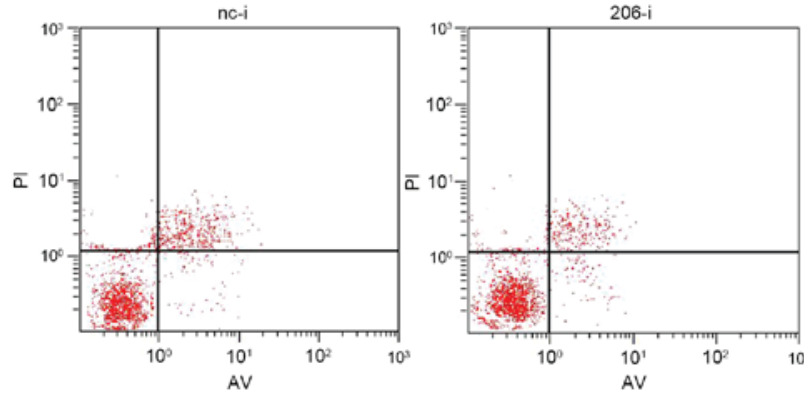
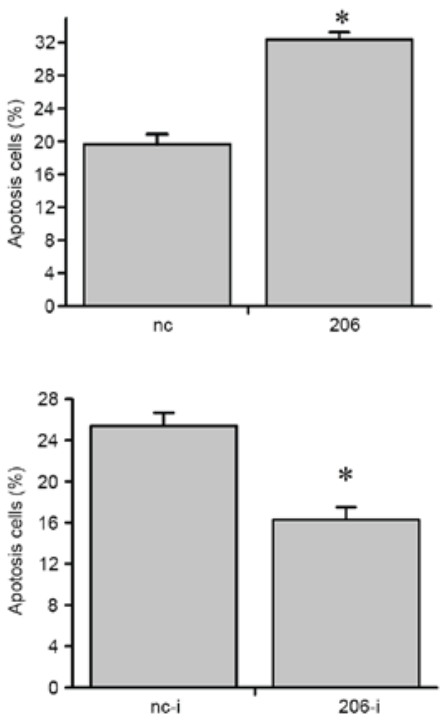

Figure 4. Effect of miR-206 on cell proliferation and apoptosis. (A) Cell proliferation in the four groups (nc, nc-I, 206 and 206-i). OD value was recorded to evaluate cell proliferation. (B) Cell apoptosis rate in the four groups. Compared with control group (nc, nc-i, respectively). * $<<0.05$. OD, optical density; AV, Annexin V; PI, propidium iodide. miR-206, microRNA 206; nc, group transfected with pcmv-nc; nc-i, group transfected with pgpu6-206; 206, group transfected with pcmv-206; 206-i, group transfected with pgpu6-206. MOD, mean optical density.

chronic obstructive pulmonary disease. miR-206 is involved in the formation of smooth muscle in the airway (31) and hypoxia-induced pulmonary hypertension due to an interaction with the hypoxia inducible factor $1 \mathrm{a} /$ four and a half limb domains 1 pathway (32).

In previous studies, the expression of miRNAs in the lungs of BPD mice was demonstrated to be different from non-BPD mice $(17,19)$. It was also reported that expression of miR-206 was lower in the plasma of BPD patients and BPD mice compared with control group (7). These results indicated that miR-206 was closely associated with BPD.

It has also been indicated that abnormal remodeling of the ECM is a hallmark feature of BPD $(3,7)$. The ECM proteins form intricate fibrillar networks, which regulate cell adhesion, migration and growth. Genes associated with ECM remodeling, including transforming growth factor- $\beta 1$ (TGF- $\beta 1$ ), tissue inhibitor of metalloproteinases 1 and collagen $1 \alpha$, were deregulated in the development of BPD (7).

FN1, as a component of the ECM, is involved in mediating a number of biological processes including cell adhesion, migration, apoptosis and signal transduction. The glycoprotein is widely distributed in blood vessel and smooth-muscle cell layers. Abnormal regulation of FN1 has been reported in several human diseases, including cancers. FN1 is able to interact with other ECM proteins, including collagen, vascular endothelial growth factor and TGF- $\beta 1$, which serve important roles in the development of BPD $(8,9)$. Furthermore, FN1 is induced by inflammation, which has been associated with causing BPD $(33,34)$. The expression of FN1 was demonstrated to increase in BPD mice and patients (7). Therefore, implying that FN1 is involved in the development of BPD.

An miRNA may regulate a variety of genes. In the present study, the gene and protein expression of FN1 was detected following the transfection of different plasmids into AECII. The results indicated that upregulation of miR-206 may inhibit the expression of FN1 in AECII. While FN1 serves a vital role in BPD, the current study may help improve the understanding of the underlying mechanism of BPD.

AECII are able to synthesize and secrete alveolar surfactants to reduce surface tension. AECII are able to differentiate into the type I alveolar epithelial cells, which are progenitor cells for the re-epithelization of impaired alveoli $(3,16,21,22)$. However, excessive proliferation and abnormal differentiation of AECII is closely associated with BPD $(3,7)$. Data from the current study indicated that miR-206 inhibited proliferation and promoted apoptosis of AECII. Therefore indicating that miR-206 may partly prevent the progression of BPD.

In conclusion, the present in vitro study suggests that miR-206 modulates numerous aspects of biology in AECII. Offering novel insights into the prevention of BPD and indicating that miR-206 may be used as a promising molecular target for BPD therapy in the future. 


\section{Acknowledgements}

The present study was supported by the National Natural Science Foundation of China (Beijing, China) (grant nos. 81270059 and 81170602 ).

\section{References}

1. Rossor T and Greenough A: Advances in paediatric pulmonary vascular disease associated with bronchopulmonary dysplasia. Expert Rev Respir Med 9: 35-43, 2015.

2. Nyp MF, Taylor JB, Norberg M and Truog WE: Impaired growth at birth and bronchopulmonary dysplasia classification: Beyond small for gestational age. Am J Perinatol 32: 75-82, 2015.

3. Popova AP: Mechanisms of bronchopulmonary dysplasia. J Cell Commun Signal 7: 119-127, 2013.

4. Jain D and Bancalari E: Bronchopulmonary dysplasia: Clinical perspective. Birth Defects Res A Clin Mol Teratol 100: 134-144, 2014.

5. Strueby L and Thébaud B: Advances in bronchopulmonary dysplasia. Expert Rev Respir Med 8: 327-338, 2014.

6. Thomas W and Speer CP: Chorioamnionitis is essential in the evolution of bronchopulmonary dysplasia-the case in favour. Paediatr Respir Rev 15: 49-52, 2014

7. Zhang X, Xu J, Wang J, Gortner L, Zhang S, Wei X, Song J, Zhang Y, Li Q and Feng Z: Reduction of microRNA-206 contributes to the development of bronchopulmonary dysplasia through up-regulation of fibronectin 1. PLoS One 8: e74750, 2013.

8. Yen CY, Huang CY, Hou MF, Yang YH, Chang CH, Huang HW, Chen $\mathrm{CH}$ and Chang HW: Evaluating the performance of fibronectin 1 (FN1), integrin $\alpha 4 \beta 1$ (ITGA4), syndecan-2 (SDC2) and glycoprotein CD44 as the potential biomarkers of oral squamous cell carcinoma (OSCC). Biomarkers 18: 63-72, 2013.

9. Soikkeli J, Podlasz P, Y in M, Nummela P, Jahkola T, Virolainen S, Krogerus L, Heikkila P, von Smitten K, Saksela O and Hölttä E: Metastatic outgrowth encompasses COL-I, FN1, and POSTN up-regulation and assembly to fibrillar networks regulating cel adhesion, migration, and growth. Am J Pathol 177: 387-403, 2010.

10. Viana Lde S, Affonso RJ Jr, Silva SR, Denadai MV, Matos D, Salinas de Souza C and Waisberg J: Relationship between the expression of the extracellular matrix genes SPARC, SPP1, FN1, ITGA5 and ITGAV and clinicopathological parameters of tumor progression and colorectal cancer dissemination. Oncology 84: 81-91, 2013.

11. Lee RC, Feinbaum RL and Ambros V: The C.elegans heterochronic gene lin-4 encodes small RNAs with antisense complementarity to lin-14. Cell 75: 843-854, 1993.

12. Wightman B, Ha I and Ruvkun G: Posttranscriptional regulation of the heterochronic gene lin-14 by lin- 4 mediates temporal pattern formation in C. elegans. Cell 75: 855-862, 1993.

13. Moreno-Moya JM, Vilella F and Simón C: MicroRNA: Key gene expression regulators. Fertil Steril 101: 1516-1523, 2014

14. Rothman AM, Chico TJ and Lawrie A: MicroRNA in pulmonary vascular disease. Prog Mol Biol Transl Sci 124: 43-63, 2014.

15. Wagner M, Vicinus B, Frick VO, Auchtor M, Rubie C, Jeanmonod P, Richards TA, Linder R and Weichert F: MicroRNA target prediction: Theory and practice. Mol Genet Genomics 289: 1085-1101, 2014.

16. Suzuki HI, Katsura A, Matsuyama $\mathrm{H}$ and Miyazono K: MicroRNA regulons in tumor microenvironment. Oncogene 34: 3085-3094, 2015.
17. Zhang X, Peng W, Zhang S, Wang C, He X, Zhang Z, Zhu L, Wang $Y$ and Feng Z: MicroRNA expression profile in hyperoxia-exposed newborn mice during the development of bronchopulmonary dysplasia. Respir Care 56: 1009-1015, 2011.

18. Zhang XQ, Zhang P, Yang Y, Qiu J, Kan Q, Liang HL, Zhou XY and Zhou XG: Regulation of pulmonary surfactant synthesis in fetal rat type II alveolar epithelial cells by microRNA-26a. Pediatr Pulmonol 49: 863-872, 2014.

19. Yang Y, Qiu J, Kan Q, Zhou XG and Zhou XY: MicroRNA expression profiling studies on bronchopulmonary dysplasia: A systematic review and meta-analysis. Genet Mol Res 12: 5195-5206, 2013.

20. Rogers LK, Robbins M, Dakhlallah D, Yang Z, Lee LJ, Mikhail M, Nuovo G, Pryhuber GS, McGwin G, Marsh CB and Tipple TE: microRNA-17 92 cluster expression is attenuated in bronchopulmonary dysplasia. Ann Am Thorac Soc (In press)

21. Sugahara K, Tokumine J, Teruya K and Oshiro T: Alveolar epithelial cells: Differentiation and lung injury. Respirology 11 (Suppl): S28-S31, 2006

22. Lee JW, Fang X, Dolganov G, Fremont RD, Bastarache JA, Ware LB and Matthay MA: Acute lung injury edema fluid decreases net fluid transport across human alveolar epithelial type II cells. J Biol Chem 282: 24109-24119, 2007.

23. Albus U: Guide for the Care and Use of Laboratory Animals. 8th edition. Washington (DC), National Academies Press (US), 2011

24. Livak KJ and Schmittgen TD: Analysis of relative gene expression data using real-time quantitative PCR and the 2(-Delta Delta C(T)) Method. Methods 25: 402-408, 2001.

25. Du F, Wu H, Zhou Z and Liu YU: microRNA-375 inhibits osteogenic differentiation by targeting runt-related transcription factor 2. Exp Ther Med 10: 207-212, 2015.

26. Zhou J, Tian Y, Li J, Lu B, Sun M, Zou Y, Kong R, Luo Y, Shi Y, Wang $\mathrm{K}$ and Ji G: miR-206 is down-regulated in breast cancer and inhibits cell proliferation through the up-regulation of cyclinD2. Biochem Biophys Res Commun 433: 207-212, 2013.

27. Li Y, Hong F and Yu Z: Decreased expression of microRNA-206 in breast cancer and its association with disease characteristics and patient survival. J Int Med Res 41: 596-602, 2013.

28. Wu J, Yang T, Li X, Yang Q, Liu R, Huang J, Li Y, Yang C and Jiang Y: Alteration of serum miR-206 and miR-133b is associated with lung carcinogenesis induced by 4- (methylnitrosamino)-1(3-pyridyl)-1-butanone. Toxicol Appl Pharmacol 267: 238-246, 2013.

29. Yang Q, Zhang C, Huang B, Li H, Zhang R, Huang Y and Wang J: Downregulation of microRNA-206 is a potent prognostic marker for patients with gastric cancer. Eur J Gastroenterol Hepatol 25: 953-957, 2013.

30. Lewis A, Riddoch-Contreras J, Natanek SA, Donaldson A, Man WD, Moxham J, Hopkinson NS, Polkey MI and Kemp PR: Downregulation of the serum response factor/miR-1 axis in the quadriceps of patients with COPD. Thorax 67: 26-34, 2012.

31. Radzikinas K, Aven L, Jiang Z, Tran T, Paez-Cortez J, Boppidi K, Lu J, Fine A and Ai X: A Shh/miR-206/BDNF cascade coordinates innervation and formation of airway smooth muscle. J Neurosci 31: 15407-15415, 2011.

32. Yue J, Guan J, Wang X, Zhang L, Yang Z, Ao Q, Deng Y, Zhu P and Wang G: MicroRNA-206 is involved in hypoxia-induced pulmonary hypertension through targeting of the HIF-1a/Fhl-1 pathway. Lab Invest 93: 748-759, 2013.

33. Fujiu K, Manabe I and Nagai R: Renal collecting duct epithelial cells regulate inflammation in tubulointerstitial damage in mice. J Clin Invest 121: 3425-3441, 2011.

34. Lumeng CN, Deyoung SM, Bodzin JL and Saltiel AR: Increased inflammatory properties of adipose tissue macrophages recruited during diet-induced obesity. Diabetes 56: 16-23, 2007. 